\title{
A QoS-aware Adaptive Mobility Handling Approach for LoRa-based IoT Systems
}

\author{
Robbe Berrevoets \\ Department of Computer Science \\ KU Leuven, Belgium \\ Email: robbe.berrevoets@student.kuleuven.be
}

\author{
Danny Weyns \\ Department of Computer Science \\ KU Leuven, Belgium \& Linnaeus University, Sweden \\ Email:danny.weyns@kuleuven.be
}

\begin{abstract}
Internet-of-Things (IoT) is an emergent paradigm that is increasingly applied in smart cities. A popular technology used in IoT is LoRa that supports long-range wireless communication. In this research, we study LoRa-based IoT systems with batterypowered end nodes that collect and communicate data to a gateway for further processing. Existing approaches in such IoT systems usually only consider stationary end nodes. We focus on systems with mobile end nodes, paving the way to new applications such as target tracking. Key Quality of Service (QoS) requirements for these settings are the reliability of the communication and energy consumption. With mobile end nodes, ensuring these QoS is challenging as the system is subject to continuous changes. In this paper, we investigate how the settings of a mobile end node impact key performance indicators for reliability and energy consumption. Based on insights obtained from extensive field experiments, we devise an algorithm that automatically adapts the settings of a mobile end node to ensure its QoS requirements for a setup with a single gateway. We then extend the algorithm to a setup with multiple gateways. We demonstrate how the algorithms achieve the QoS requirements of a mobile end node in a concrete IoT deployment.
\end{abstract}

\section{Introduction}

The Internet of Things (IoT) is an emerging paradigm that aims at giving objects around us the ability to interact and cooperate with each other and users to accomplish various tasks. IoT is widely considered as a key enabler for realizing smart cities. According to their estimates, Ericsson expects that by 2023, 20 billion objects will be part of the IoT [1].

An important class of IoT systems consists of small battery-powered computer elements (end nodes) that are equipped with sensors and actuators and communicate wirelessly. A typical use case is an IoT setup with end nodes that gather local data about the environment and relay this data to a gateway from where the data is processed for users [2], [3].

Popular communication technologies used in IoT are LoRa [4] and LoRaWAN [5] that builds on LoRa technology. LoRa supports long range wireless communication using a novel modulation scheme. LoRaWAN provides support for network routing, security, among other features. In this research, we study LoRa-based IoT systems with battery-powered end nodes that collect and communicate data to one or more gateways for further processing.

IoT is applied in various domains, e.g., home automation, health monitoring, smart manufacturing, precision agriculture, and area surveillance, see for example [6]. In most of the current applications end nodes are stationary. However, many application domains would benefit from mobile end nodes, such as habitat monitoring, logistics, and product life-cycle management [7], [8]. While mobility in IoT systems gives rise to new opportunities it also introduces significant challenges.

In general, stakeholders of IoT systems have various Quality-of-Service (QoS) requirements, such as low energy consumption, low packet loss and latency, etc. The priority of these requirements depends on specifics of the domain. Mobility of end nodes typically introduces additional requirements, such as localization, navigation, coverage, etc. [9]. In this paper we focus on two QoS requirements that are generally considered as key for battery-powered wireless IoT systems: reliability of communication and energy consumption of end nodes [10], [11]. Reliability depends on the packets that get lost during transmission, while the energy consumed by battery-powered end nodes affects the life time of the network.

Achieving high reliability and low energy consumption in IoT systems is challenging as these requirements are conflicting (higher transmission power typically results in a lower packet loss), see e.g., [12]. With mobile nodes, ensuring the QoS requirements becomes even more challenging [4]. Moving end nodes are subject to changing conditions in the environment that affect their connectivity with the gateways. As these conditions may be difficult to predict, one approach to ensure the required QoS is by applying conservative settings based on worst-case conditions. Such an approach, which is often used in practice [13], may achieve high reliability, but at the cost of high energy consumption.

To address the problem of achieving the required QoS in mobile LoRa-based IoT Systems we apply an adaptive approach that tracks the environment conditions and automatically adapts the node settings to achieve the required QoS. The contributions are: (1) we determine the relationship between the settings of a mobile LoRa-based end node and key performance indicators for reliability and energy consumption; (2) we devise a novel algorithm that allows a mobile node to adapt its settings autonomously to ensure its QoS requirements in a setup with one or more gateways. We demonstrate the effectiveness of the algorithm in a practical IoT deployment.

The remainder of this paper is structured as follows. In Section 2 we provide the necessary background on LoRa. Section 3 presents the research questions and the research approach we have applied to answer them. In Section 4 we study the impact of settings of a mobile end node on reliability and energy consumption. Section 5 presents the 
adaptation algoritms for a mobile end node to achieve its QoS requirements, with a single gateway and multiple gateways. In Section 6, we evaluate the algorithms for a practical IoT setup. Section 7 discusses related efforts. Finally, we draw conclusions and outline plans for future work in Section 8.

\section{Background}

LoRa offers a wireless modulation scheme that can be used to create long distance communication links. Technically, LoRa is based on chirp spread spectrum modulation, which is similar to Frequency Shifting Keying, but with significantly increased communication range. ${ }^{1}$ Chirp spectrum uses the entire allocated bandwidth to broadcast a signal. This approach is resistant to multi-path fading and the Doppler effect, even when operating at low power [4]. These features make LoRa particularly useful in mobile and urban settings.

Several parameters settings are available to customize the LoRa communication. We discuss two parameters relevant to this research study: spreading factor and power setting.

The spreading factor (SF) defines the time on air of a communication (technically SF defines the duration of the chirp). LoRa uses spreading factors from 7 (shortest time on air) to 12 (longest time on air). An increment in spreading factor doubles the time on air to transmit the same amount of data and results in an increase in communication range. In our experiments, we consider SF from 7 to 12 .

The power setting (POW) of an end node determines the energy that is used for transmitting packets, which is the dominating factor of energy used by end nodes [14]. In LoRa, POW can be set in a range, e.g., -3 to 15 . In our experiments, we use POW low (-3), medium (6), and high (15).

Additional factors important for a mobile LoRa network are the speed and direction of end nodes [15], and the conditions in the environment of the network [16], [17], [18]. In our experiments, we keep the speed of end nodes stable at approximately $1.6 \mathrm{~m} / \mathrm{s}$ (human walking speed), while moving on a straight line. The conditions in the environment of the network are kept as similar as possible.

The parameter settings of the mobile end node will affect several performance indicators of the IoT system. We discuss the indicators that are relevant to this research: energy consumption, signal-to-noise ratio, received signal strength indication, and packet error rate.

The energy consumption $(E C)$ determines the amount of energy consumed by of an end node and is defined as:

$$
E C=\sum_{i \in \text { packets }} \text { SupplyCurrent }_{i} * t_{i}(\text { Transmitting })
$$

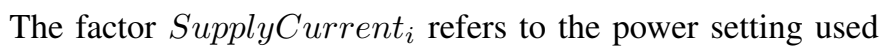
by the end node to transmit packet $i$, while $t_{i}$ (Transmitting) refers to the time used by the radio to transmit packet $i$.

The signal-to-noise-ratio (SNR) is defined as the ratio of power of the signal to the power of the noise, usually expressed in decibels $(\mathrm{dB})$. A ratio higher than $1: 1(>0 \mathrm{~dB})$ indicates that the signal is stronger as the noise.

$$
S N R_{d B}=10 * \log _{10}\left(\frac{P_{\text {signal }}}{P_{\text {noise }}}\right)
$$

1. www.semtech.com/uploads/documents/an1200.22.pdf
The received signal strength indicator (RSSI) is an indication of the power level being received by the receiver; the higher the RSSI, the stronger the signal. The numbers however are arbitrary. There is no standardized relationship of any particular physical parameter to the RSSI reading.

The packet error rate (PER) is an indicator for the reliability of the communication:

$$
\text { PER }=1-\frac{\# \text { packets correctly received }}{\# \text { packets sent }}
$$

As wireless communication is inherently sensitive to errors, stakeholders have to determine an acceptable PER for the application at hand. In our experiments, we set PER at $10 \%$, but, this level is flexible and can be changed as needed.

In addition to PER, we use the notion of connected region as an indicator for reliability, which is defined as a region around a receiver (gateway) where the PER of a sender (end node) is below a given threshold [19]. Although wireless communication is often anisotropic, connected regions based on a low PER threshold show a more isotropic behaviour allowing to measure a connected region one-dimensionally. In our experiments, we set the threshold for connected regions to a PER level of $10 \%$.

\section{Research Questions and Methodology}

The goal of this research is to enable a mobile LoRa end node to adapt dynamically its setting to achieve its QoS requirements, in particular reliability and energy consumption. To that end, we study the following research questions:

RQ1: What is the impact of different settings of a mobile LoRa end node (SF, POW) on key performance indicators for reliability and energy consumption (EC, SNR, RRSI, PER)?

RQ2: How to adapt the settings of a mobile end node dynamically to achieve its QoS requirements with a single gateway?

RQ3: How to adapt the settings of a mobile end node dynamically to achieve its QoS requirements with multiple gateways?

To answer these research questions, we defined an empirically grounded research approach, shown in Figure 1.

Step 1 defines the research questions (see above) by consulting the literature. In step 2, an empirical IoT test setup is defined. This setup is then used in step 3 to empirically determine the impact of different settings of a mobile end node on performance indicators for QoS. The insights from these experiments are used to define an algorithm in step 4 that allows a mobile node to adapt its settings and achieve its QoS requirements with a single gateway. In the fifth and final step, the algorithm is extended for multiple gateways. The algorithms are evaluated with the IoT deployment.

\section{Impact of Settings of a Mobile End Node on Key Performance Indicators}

In this section, we explain steps 2 and 3 of the research approach, aiming to answer the first research question.

Our goal is to investigate how different node settings (SF and POW) and distance between the sender (moving end node) and the receiver (gateway) impact key performance indicators for reliability and energy consumption (EC and PER). As 


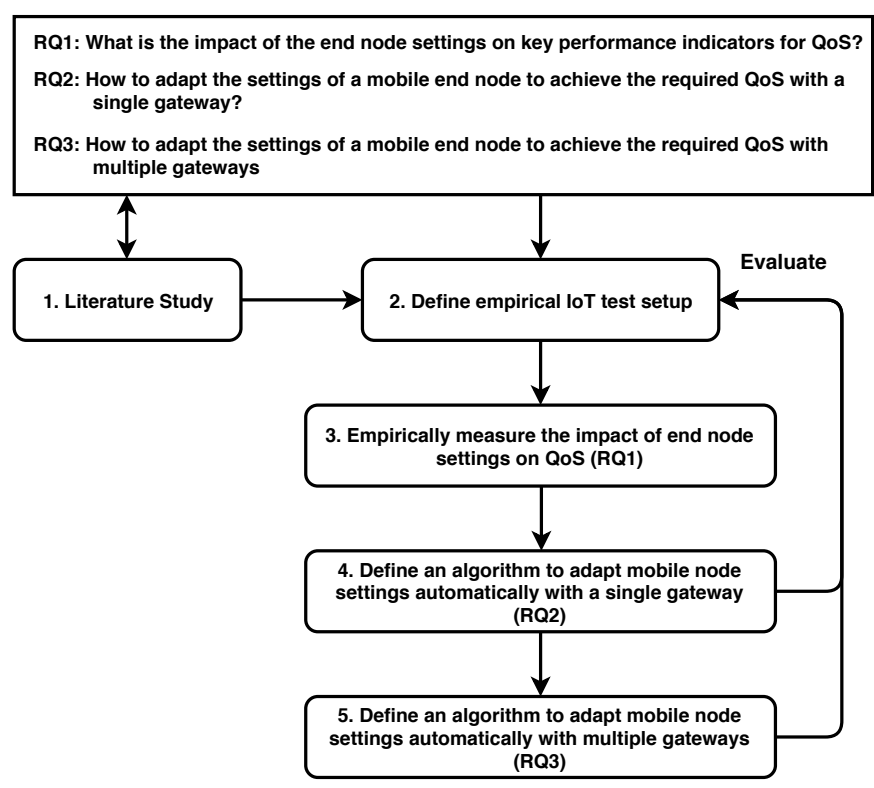

Figure 1: Schematic overview of the research approach

energy consumption and reliability are conflicting requirements, a trade-off has to be made between these two QoS requirements. With answering RQ1, we aim to understand how to make this trade-off. To that end, we need to understand the relationship between end node settings, mobility, and the performance indicators of the QoS requirements.

\subsection{Experimental Setup}

Figure 2 shows a typical network setup with mobile end nodes. LoRa allows end nodes to communicate directly with gateways (forming a star topology). Packets sent by end nodes can be received by the gateways that are within communication range (e.g., end node 1 is currently in range of Gateway 1 and Gateway 2). Gateways relay the packets they receive to the application backend (which may be hosted in the Cloud).

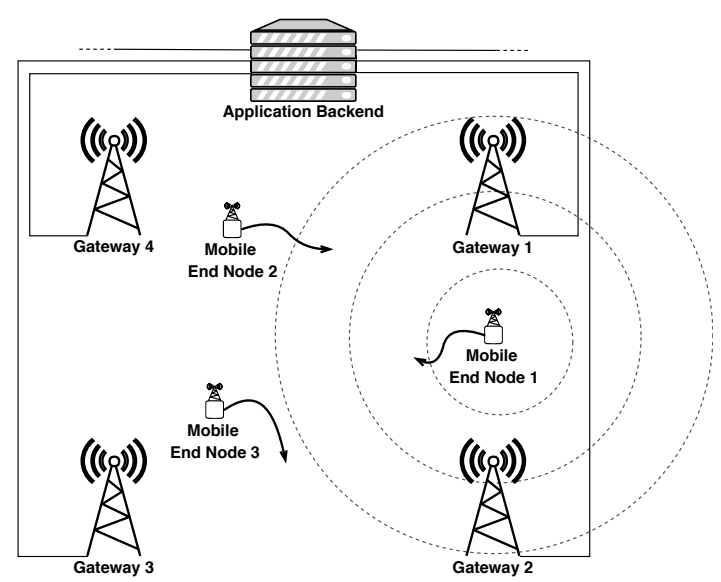

Figure 2: Network architecture for a mobile LoRa network

For the experiments, we have set up an experimental IoT system shown in Figure 3. The white line shows the trajectory that the end node follows. Gateways GW1 and GW2 are positioned at $3.21 \mathrm{~km}$ from each other, both are located on top of a building. These buildings are located in an industrial area, thus the environment is similar across the trajectory.

A gateway consists of a LoRa concentrator (IMST ic $880 \mathrm{a}^{2}$ ), a GPS module (Adafruit Ultimate GPS $^{5}$ ) and a microcontroller $\mu C$ (Raspberry Pi 3 model $\mathrm{B}^{6}$ ) as shown in Figure 4. The LoRa concentrator receives the LoRa messages; the source code is developed by Semtech ${ }^{3}$. The microcontroller fetches the received packets and relevant metadata from the LoRa concentrator. This information is transmitted to the application backend via a TCP connection. The application backend processes the data received from all gateways to provide the application functionality for the users. The GPS module determines the location of the gateway. A box containing the components of a gateway is placed on a free location at the top of a high building (around $25 \mathrm{~m}$ high).

An end node consists of a LoRa transmitter (Microchip Technology Mote ${ }^{4}$ ), a GPS module (Adafruit Ultimate GPS ${ }^{5}$ ), and a microcontroller $\mu C$ (Raspberry Pi 3 model $\mathrm{B}^{6}$ ) as shown in Figure 4. The LoRa transmitter is responsible for the LoRa communication. We used a bandwidth of $125 \mathrm{kHz}$, with a carrier at 868.1 Mhz. The microcontroller decides when to send messages, determines the content of the packets, reconfigures the LoRa parameters, and logs the GPS coordinates. The components of the end node are assembled in a box that can be carried by a person while moving.

LoRa Parameters. The LoRa transmitter offers 18 power settings ( -3 to 15$)$ and 6 spreading factor settings (7-12). We only examined POW $-3,6$ and 15 and SF $7-8$ and $10-12 .^{7}$ In the next sections, we present the results of the experiments that assess the impact of these settings (POW, SF) on the key performance indicators of reliability and energy consumption. During the experiments, an end node moved from GW1 to GW2 (for these experiment only GW1 is active) at $1.6 \mathrm{~m} / \mathrm{s}$. Table 1 gives an overview of the number of measurements performed per setting. All graphs in the paper are smoothed for the measurements per setting, unless mentioned differently.

\begin{tabular}{|c|c|c|c|}
\hline \multirow{2}{*}{ SF } & \multicolumn{3}{|c|}{ POW } \\
\cline { 2 - 4 } & $\mathbf{- 3}$ & $\mathbf{6}$ & $\mathbf{1 5}$ \\
\hline $\mathbf{7}$ & 1363 & 1363 & 1363 \\
\hline $\mathbf{8}$ & 1363 & 1363 & 1363 \\
\hline $\mathbf{1 0}$ & 3152 & 3152 & 3152 \\
\hline $\mathbf{1 1}$ & 2226 & 2226 & 2226 \\
\hline $\mathbf{1 2}$ & 1921 & 1921 & 1921 \\
\hline
\end{tabular}

TABLE 1: Number of measurements over the trajectory

\subsection{Reliability}

We measured the impact of the end node settings on the reliability using the notion of connected region. Recall that we defined a connected region as a region around the gateway where the PER of the end node is below $10 \%$.

Power Setting. Figure 5a shows the evolution of the PER when the end node moves along the trajectory for spreading factor 12 and different power settings. It is clear that a change

2. www.wireless-solutions.de/products/radiomodules/ic880a.html

3. www.github.com/Lora-net/lora_gateway

4. www.microchip.com/RN2483LoRaMote4233989

5. www.adafruit.com/product/746

6. www.raspberrypi.org/products/raspberry-pi-3-model-b/

7. Due to a problem with an antenna, the test results for SF 9 are omitted. 


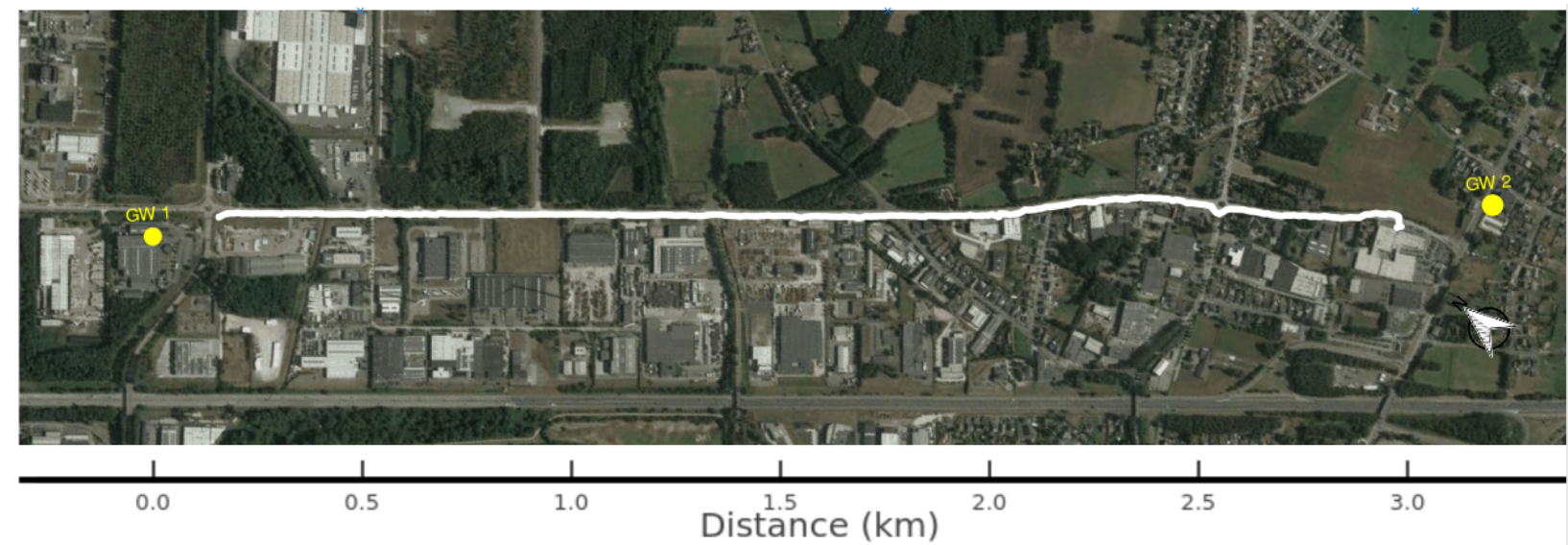

Figure 3: Experiment setup

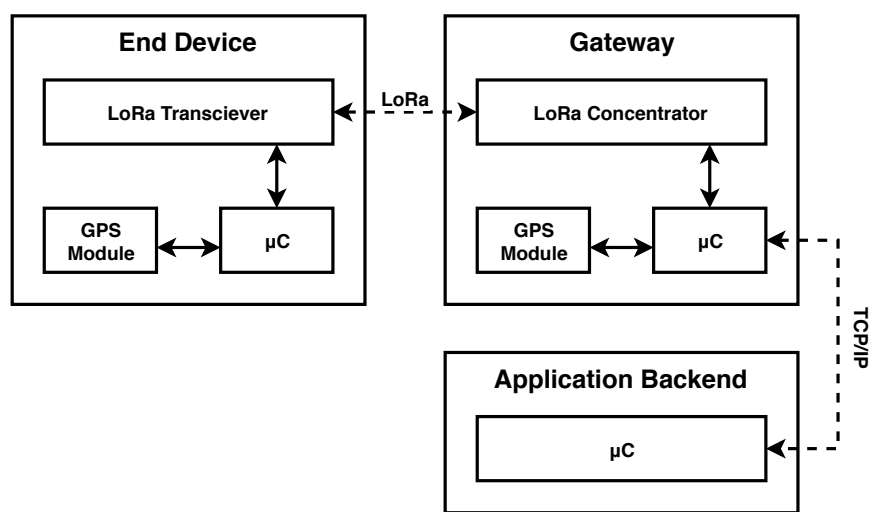

Figure 4: Component diagram with one gateway

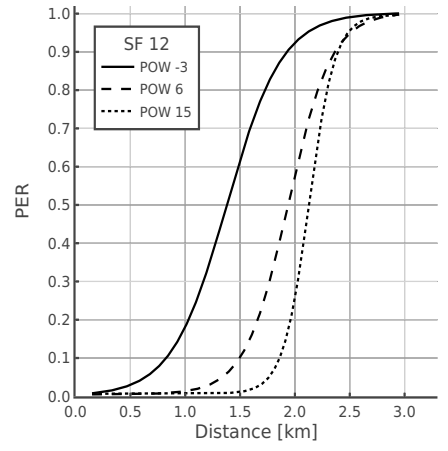

(a)

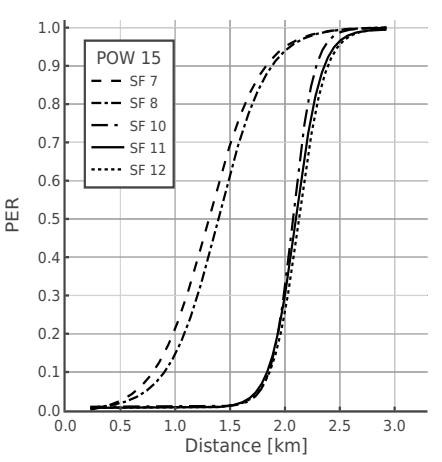

(b)
Figure 5: Evolution of PER along trajectory for: (a) SF 12 with different POW; (b) POW 15 with different SF.

in the power setting has an effect on PER-distance curve. Table 2 shows the radius of connected regions (in $\mathrm{km}$ ) for the different end node settings, allowing a quantitative comparison of the reliability between the settings. Table 3 shows that a POW increase from -3 to 6 gives an average increase of $59 \%$ in connected region radius, while an increase from 6 to 15 gives an average increase of $27 \%$ in connected region radius.

Spreading Factor. Figure $5 \mathrm{~b}$ shows the evolution of the PER along the trajectory for different spreading factors and power setting 15 . We notice that the PER-Distance curves

\begin{tabular}{|c|c|c|c|}
\hline \multirow{2}{*}{ SF } & \multicolumn{3}{|c|}{ POW } \\
\cline { 2 - 4 } & $\mathbf{- 3}$ & $\mathbf{6}$ & $\mathbf{1 5}$ \\
\hline $\mathbf{7}$ & 0.46 & 0.58 & 0.80 \\
\hline $\mathbf{8}$ & 0.43 & 0.74 & 0.90 \\
\hline $\mathbf{1 0}$ & 0.90 & 1.43 & 1.83 \\
\hline $\mathbf{1 1}$ & 0.94 & 1.49 & 1.82 \\
\hline $\mathbf{1 2}$ & 0.83 & 1.50 & 1.86 \\
\hline
\end{tabular}

TABLE 2: Connected region radius $(\mathrm{km})$ for all settings

\begin{tabular}{|c|c|c|c|c|c|c|}
\hline \multirow{2}{*}{ POW } & \multicolumn{6}{|c|}{ SF } \\
\cline { 2 - 7 } & $\mathbf{7}$ & $\mathbf{8}$ & $\mathbf{1 0}$ & $\mathbf{1 1}$ & $\mathbf{1 2}$ & $\overline{\mathbf{x}}$ \\
\hline $\mathbf{- 3 ~ t o ~ 6}$ & $+27 \%$ & $+70 \%$ & $+59 \%$ & $+57 \%$ & $+81 \%$ & $+59 \%$ \\
\hline 6 to 15 & $+36 \%$ & $+22 \%$ & $+30 \%$ & $+24 \%$ & $+24 \%$ & $+27 \%$ \\
\hline
\end{tabular}

TABLE 3: Connected region radius for increasing power settings per spreading factor setting ( $\bar{x}$ denotes the average)

are clustered for SF 7-8 and SF 10-12. Table 4 shows that only a change between clusters, from SF 8 to SF 10, has a significant effect on the average connected region radius. Due to the lack of test results for SF 9, it is not clear whether this PER-distance curve belongs to one of the clusters or lays between them. In the latter case, a more smooth transition of connected region radius would be available.

\begin{tabular}{|c|c|c|c|c|}
\hline \multirow{2}{*}{ SF } & \multicolumn{4}{|c|}{ POW } \\
\cline { 2 - 5 } & $\mathbf{- 3}$ & $\mathbf{6}$ & $\mathbf{1 5}$ & $\overline{\mathbf{x}}$ \\
\hline $\mathbf{7}$ to 8 & $-5 \%$ & $+27 \%$ & $+13 \%$ & $+11 \%$ \\
\hline $\mathbf{8}$ to 10 & $+107 \%$ & $+92 \%$ & $+106 \%$ & $+102 \%$ \\
\hline $\mathbf{1 0}$ to 11 & $+5 \%$ & $+4 \%$ & $+1 \%$ & $+3 \%$ \\
\hline $\mathbf{1 1}$ to 12 & $-12 \%$ & $+1 \%$ & $+1 \%$ & $-3 \%$ \\
\hline
\end{tabular}

TABLE 4: Impact on connected region radius for different spreading factors and different power settings

\subsection{Energy Consumption}

Now we look at the impact of the settings of the mobile end node on its energy consumption.

Power Setting. Formula (1) shows that the energy consumption is proportional to the supply current that is determined by the actual power setting. The datasheet of the Microchip Technology Mote provides the supply currents for the different power settings. Table 5 shows how increases of the power setting results in increasing energy consumption. 


\begin{tabular}{|c|c|}
\hline POW & Increase energy consumption \\
\hline $\mathbf{- 3}$ to 6 & $+59 \%$ \\
\hline $\mathbf{6}$ to 15 & $+41 \%$ \\
\hline
\end{tabular}

TABLE 5: Energy consumption for increase of POW

Spreading Factor. The spreading factor affects the energy consumption as well, as a higher spreading factor increases the time on air of the communication (Formula 1). Table 6 shows how increases of the spreading factor results in increasing energy consumption of an end node.

\begin{tabular}{|c|c|}
\hline SF & Increase energy consumption \\
\hline $\mathbf{7}$ to 8 & $+75 \%$ \\
\hline $\mathbf{8}$ to 10 & $+220 \%$ \\
\hline $\mathbf{1 0}$ to 11 & $+82 \%$ \\
\hline 11 to 12 & $+83 \%$ \\
\hline
\end{tabular}

TABLE 6: Energy consumption for increase of SF

Summary. The test results show that increasing power setting and spreading factor result in larger connected region radius and thus better reliability. However, the increase in energy consumption is lower for an increase of power settings compared to spreading factors, while the effect on reliability is better for an increase of power settings compared to spreading factor. Hence, if the settings of an end node do not satisfy the reliability requirements, the end node should first try to increase the power setting (giving the most significant gain of reliability). If the maximum power setting is reached, the end node can try to increase the spreading factor. On the other hand, if the current settings satisfy the reliability requirements, to save energy, the end node may try to reduce the spreading factor setting (as this has the least impact on reliability, but gives the most energy gain). If the minimum spreading factor is reached, the end node may try to reduce the power setting.

\section{Adaptation Algorithms}

In this section, we explain steps 4 and 5 of the research approach, aiming to answer the second and third research question. Concretely, our goal is to solve the problem of how to adapt the settings of a mobile end node such that the end node remains in a connected region (achieving the reliability requirement), while minimising consuming energy (achieving the energy consumption requirement).

To achieve this goal, we apply self-adaptation [20], [21]. Self-adaptation aims at achieving or maintaining the qualities of a software system that operates under uncertain conditions [22], [23]. To that end, changes in the system and its environment are monitored at runtime and based on the required quality goals, the system adapts itself dynamically to realize the goals (or degrade gracefully) [24]. Due to the specific characteristics of mobile IoT systems, achieving the required quality goals requires a novel adaptation solution. In particular, while moving, end nodes can cross the border of connected regions (based on the current settings). Hence, solving the adaption problem requires a mechanism that allows determining when an end node is entering or leaving a connected region. We start with solving this basic problem. Then we present a solution for the adaption problem of an end node, first for a single gateway, then with multiple gateways.
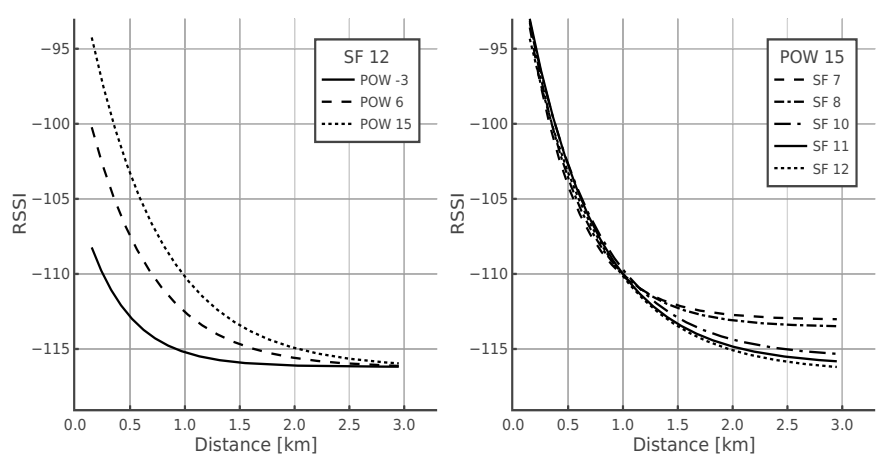

(a)

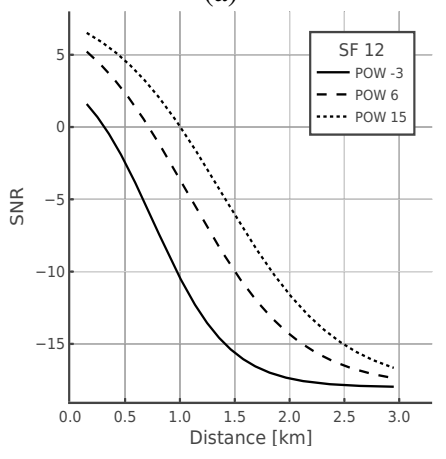

(c)

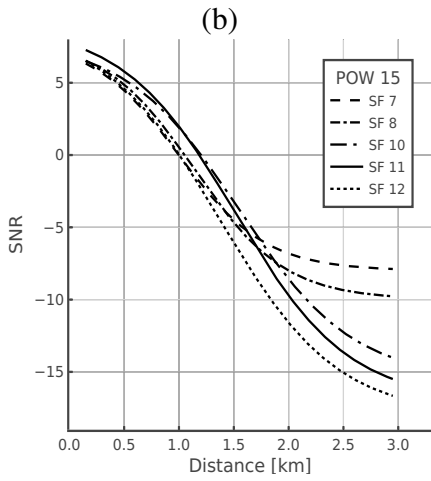

(d)
Figure 6: Evolution RSSI along trajectory for: (a) SF 12 and different power settings; (b) POW 15 and different spreading factors. Evolution SNR for: (c) SF 12 and different power settings; (d) POW 15 and different spreading factors.

\subsection{Identifying the Borders of a Connected Region}

We start with the problem of how to detect when an end node leaves/enters a connected region. A simple solution would be to equip the end node with a GPS module and make a coverage map of the configurations around the gateways. This approach requires a costly coverage analysis, and the continuous use of a GPS module drains substantial energy from the end node. Therefore, we explored other mechanisms. Baccour et al. [19] suggest using RSSI and SNR to determine the border of connected regions. The RSSI and SNR of packet communication are measured at the gateway, requiring no energy from end nodes. Hence, we examined the link between RSSI and SNR on the one hand, and PER on the other hand. To that end, the RSSI and SNR for each packet received at the gateway during the experiments explained above (when the end node moves from GW1 to GW2 and back) were captured.

Leaving a Connected Region. Figure 6 shows the evolution of RSSI and SNR in function of the distance between the end node and gateway for different settings of the end node. Figures $6 \mathrm{a}$ and $6 \mathrm{c}$ show that the power setting has an effect on both RSSI and SNR. The spreading factor on the other hand has little effect on the RSSI and SNR, as shown in Figure $6 \mathrm{~b}$ and Figure 6d. To determine whether RSSI and/or SNR of packets are good indicators to detect when an end node leaves a connected region, we plotted PER as a function of RSSI and SNR. Figure 7a and 7c show that for a particular setting of the spreading factor, the power setting has little or no effect on the PER-RSSI and PER-SNR curves. On the 

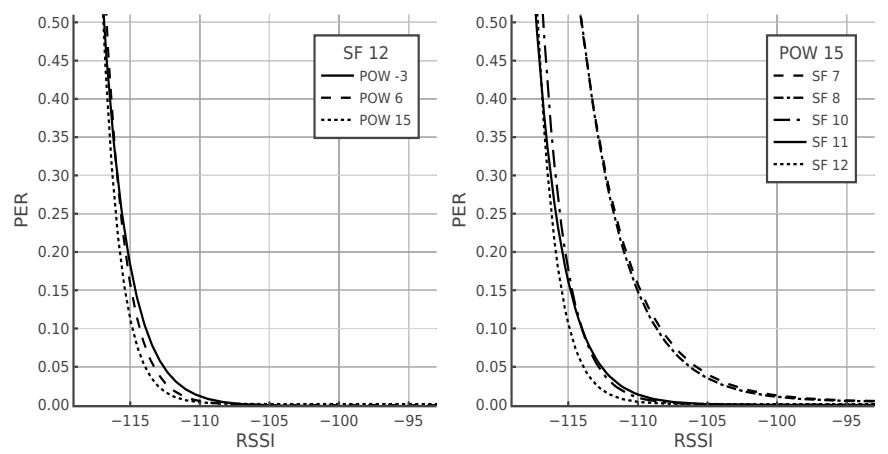

(a)
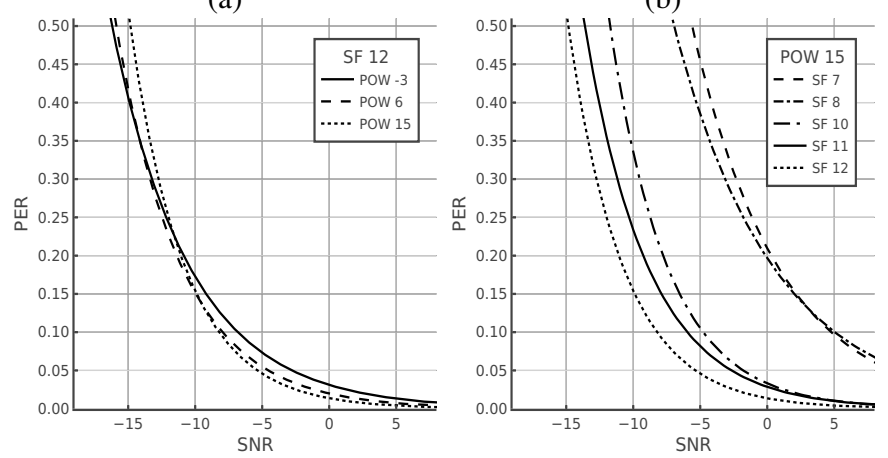

(c)

(d)

Figure 7: PER versus RSSI for: (a) SF 12 and different power settings; (b) POW 15 and different spreading factors. PER versus SNR for: (c) SF 12 and different power settings; (d) POW 15 and different spreading factors.

other hand, Figure $7 \mathrm{~b}$ and $7 \mathrm{~d}$ show that for a particular power setting, each spreading factor corresponds to a specific PERRSSI and PER-SNR curve. Thus, both SNR and RSSI provide an indication of the reliability of the communication between the end node and the gateway and as such can be used to determine when an end node leaves a connected region.

Entering a New Connected Region. When an end node moves towards a gateway, the current settings will normally not decrease the reliability of the communication. On the contrary, at some point, the current settings of power and/or spreading factor may be relaxed, while maintaining the required reliability (boiling down to entering a new connected region). To determine when an end node enters a new connected region, we need to know the effect of a change in power setting and spreading factor on the RSSI and SNR. As explained above, a change in power setting has a significant effect on the RSSI and SNR (Figures 6a and 6c), while the spreading factor has only a small effect (Figures $6 \mathrm{~b}$ and 6d). In relation to PER, each spreading factor corresponds to a specific PER-RSSI and PER-SNR curve indifferent to the power setting (Figure 7). Hence, the current values of RSSI and SNR, together with the known effects of a change of settings on RSSI and SNR and the estimated impact of these on the PER (PER-RSSI/SNR curves) allow determining whether an end node enters a new connected region.

Summary. To determine the border of a connected region, both RSSI and SNR can be used as indicator. However, Figures $7 \mathrm{a}$ and $7 \mathrm{~b}$ show a steep increase in PER in a small range of RSSI. Furthermore, the experiments show that the measured RSSI values are subject to significant variance in this RSSI range. ${ }^{8}$ In the area of the steep increase of RSSI, this variance will have a huge effect on the estimated PER and consequently on the decision whether an end node crosses the border of a connected region. Figure $7 \mathrm{c}$ and $7 \mathrm{~d}$ show that the PER estimation based on SNR is more robust to variance of the SNR measurements. Therefore, we only consider SNR as indicator for determining the border of a connected region.

\subsection{Preliminaries Adaptation Algorithms}

Deployment. In principle, the adaption algorithm can be deployed on one of the three components of the network architecture: end node, gateway, or application backend. As end nodes lack the required information to make adaptation decisions (e.g., SNR is measured at the gateways), they are not a suitable option. Thus either the gateways decide locally when end nodes should change their settings, or the gateways relay the necessary data to the application backend that makes the adaptation decisions. A problem with gateways making adaptation decisions is that they have only local information, potentially causing conflicting adaptation decisions. The application backend can leverage the data it obtains from all gateways and make optimal adaptation decisions. Hence, the adaptation algorithm is deployed at the application backend.

Required Data. To allow the application backend to make adaptation decisions, it maintains the following data:

- For each end node: (i) the desired PER and (ii) the recent SNR history of received packets per gateway;

- The SNR-PER function per SF;

- The SNR-POW change functions;

- The SNR-SF change functions.

End nodes have to determine their desired PER level and inform the application backend, typically when they start operating. The gateways measure the SNR of packets they receive and forward the data to the application backend. The application backend keeps track of the history of SNR values and groups them per gateway. The SNR-PER function for each SF is experimentally determined (see Figure 7d). Two additional functions are used for decision making that are derived from the data of the graphs show in Figure 6. The SNR-POW change function describes the effect on SNR when changing the POW setting. Figure 8a shows the SNR-POW change function for a change from POW 15 to POW 6. For example, consider a location where SNR is initially -4 with a POW setting of 15 (dotted lines). If the end node changes POW to 6 the SNR will be reduced to -8.2. The SNR-SF change function (an example is shown in Figure $8 \mathrm{~b}$ ) similarly describes the effect on the SNR when changing the SF setting.

\subsection{Adaptation Algorithm with Single Gateway}

Algorithm 1 shows the adaptation procedure with a single gateway. The algorithm starts with extracting relevant data when a packet arrives at the application backend (lines 2 to $5)$, i.e., the identifier of the end node that has sent the packet $(E N)$, the current settings of the end node $(S F, P O W)$, and

8. E.g., for a setting SF 12 and POW 15 we measured on average a variance of $[-4.9,+4.9]$ for RSSI values that change between $[-118,-112]$ 


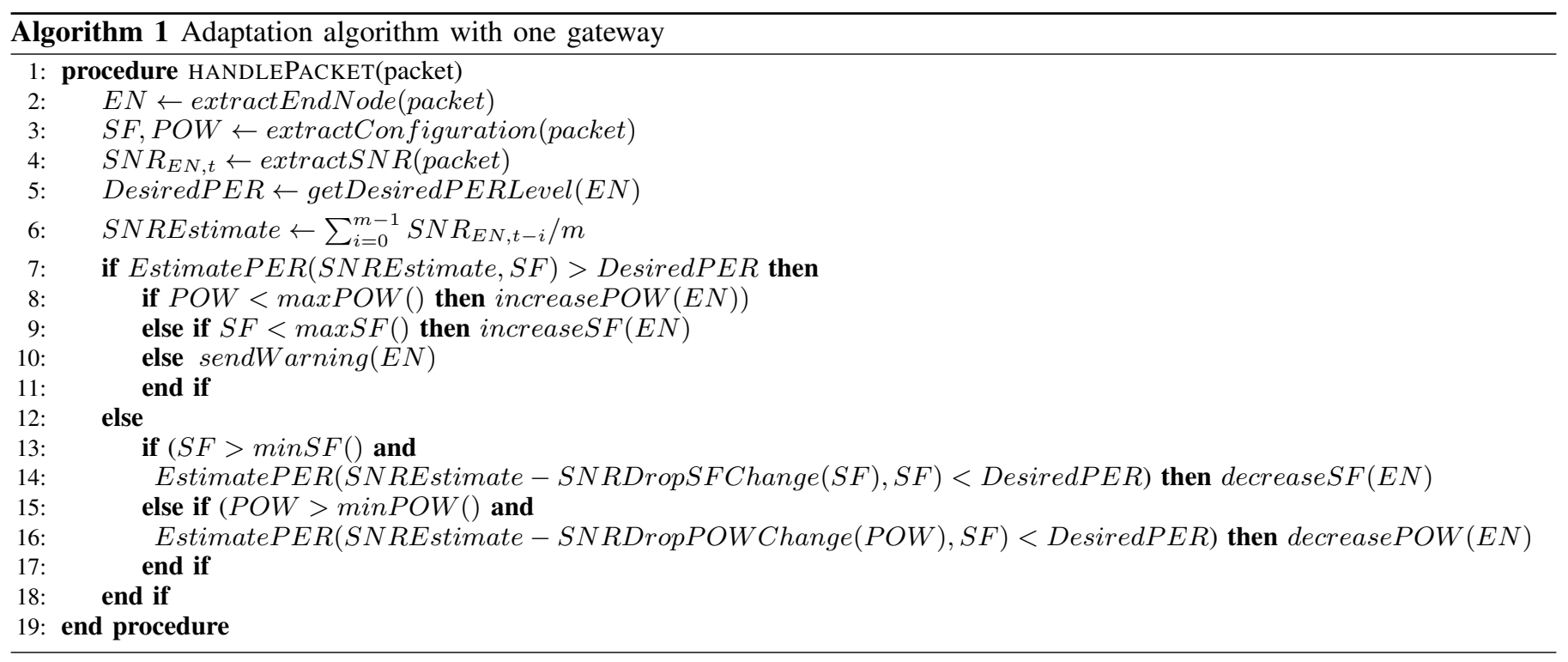

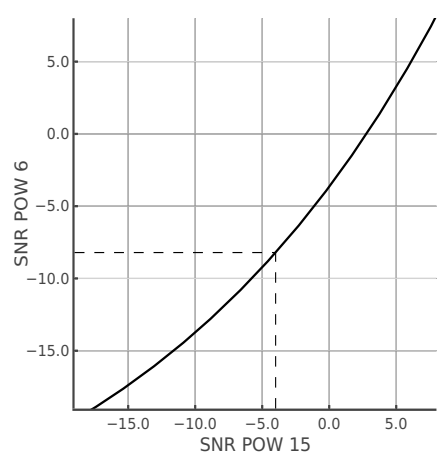

(a)

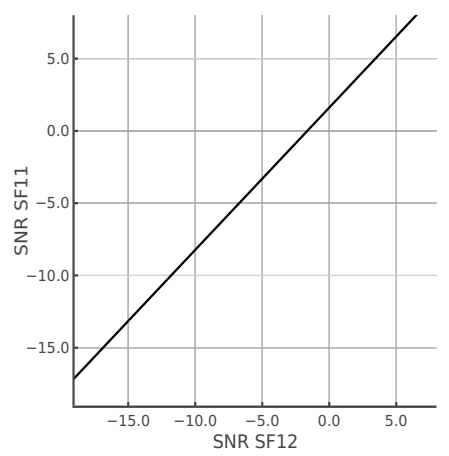

(b)
Figure 8: Change functions (a) SRN-POW, (b) SRN-SF.

the SNR measurement of the packet $\left(S N R_{E N, t}\right)$ that is added to the SNR history of the end node. Finally, the desired PER level of the end node that sent the packet is fetched from the data associated with that end node.

Next, the algorithm calculates an estimate for the SNR (line 6). In our research, we apply a windowed moving average that takes the average of the last $m$ SNR measurements associated to the end node. However, other more advanced approaches can be plugged in to estimate the SNR.

Subsequently, the algorithm determines whether or not a change of the end node settings is required. First, the algorithm tests if the end node is leaving the connected region (lines 7 to 11). Using the SNR-PER function of the current SF and the SNR estimate, the algorithm calculates the estimated PER. If the estimated PER is above the desired PER level, the settings need to be adapted. The algorithm will try to increase the power setting, if this setting achieved its maximum, it will increase the spreading factor. If both POW and SF have reached their maximum value, a warning is generated. Second, if the estimated PER is below the desired level, the algorithm tests whether the end node is entering a new connected region (lines 12 to 17). If the PER estimate after a change of settings remains lower as the desired PER, the algorithm can relax the current configuration, i.e. the spreading factor or the power setting can be decreased in that order.

\subsection{Adaptation Algorithm with Multiple Gateways}

Algorithm 2 shows the adaptation procedure for a setup with multiple gateways. When the application backend receives a packet it listens during a certain time window for duplicates that may be relayed by other gateways. Next, all duplicate packets are fed to the algorithm.

The structure of the algorithm for multiple gateways is similar as for a single gateway. The main difference is the way the PER of the end node is estimated as the algorithm can now use data from all gateways that relayed the same packet. In particular, an estimate for the PER at each gateway is determined and used to determine an estimate of the global PER level (based on probability of a parallel system):

$$
P E R=\prod_{i \in \text { gateways }} P E R_{i}
$$

The gateway best serving the end node is the one that achieves the lowest PER level. This gateway is used to send updates to the end node if necessary.

Similar to the algorithm for one gateway, the need for an increase in power setting or spreading factor is determined by comparing the estimated PER with the desired PER. When determining whether the end node settings can be relaxed (decrease of SF or POW), the algorithm only looks at the gateway best serving the end node, as a relaxation of the end node settings may affect the PER levels at all gateways, potentially causing a large global effect (see Equation 4). By considering only the best gateway and making sure the PER at this gateway stays below the desired PER, the global PER level will never exceed the desired PER level.

\section{Evaluation}

We now evaluate the two adaptation algorithms. The QoS requirements for both experiments are the same:

R1 Keep the PER of the end node below 10\%;

R2 Subject to R1, minimize the EC of the end node.

We start with the evaluation with one gateway followed by multiple gateways. We conclude with threats to validity. 


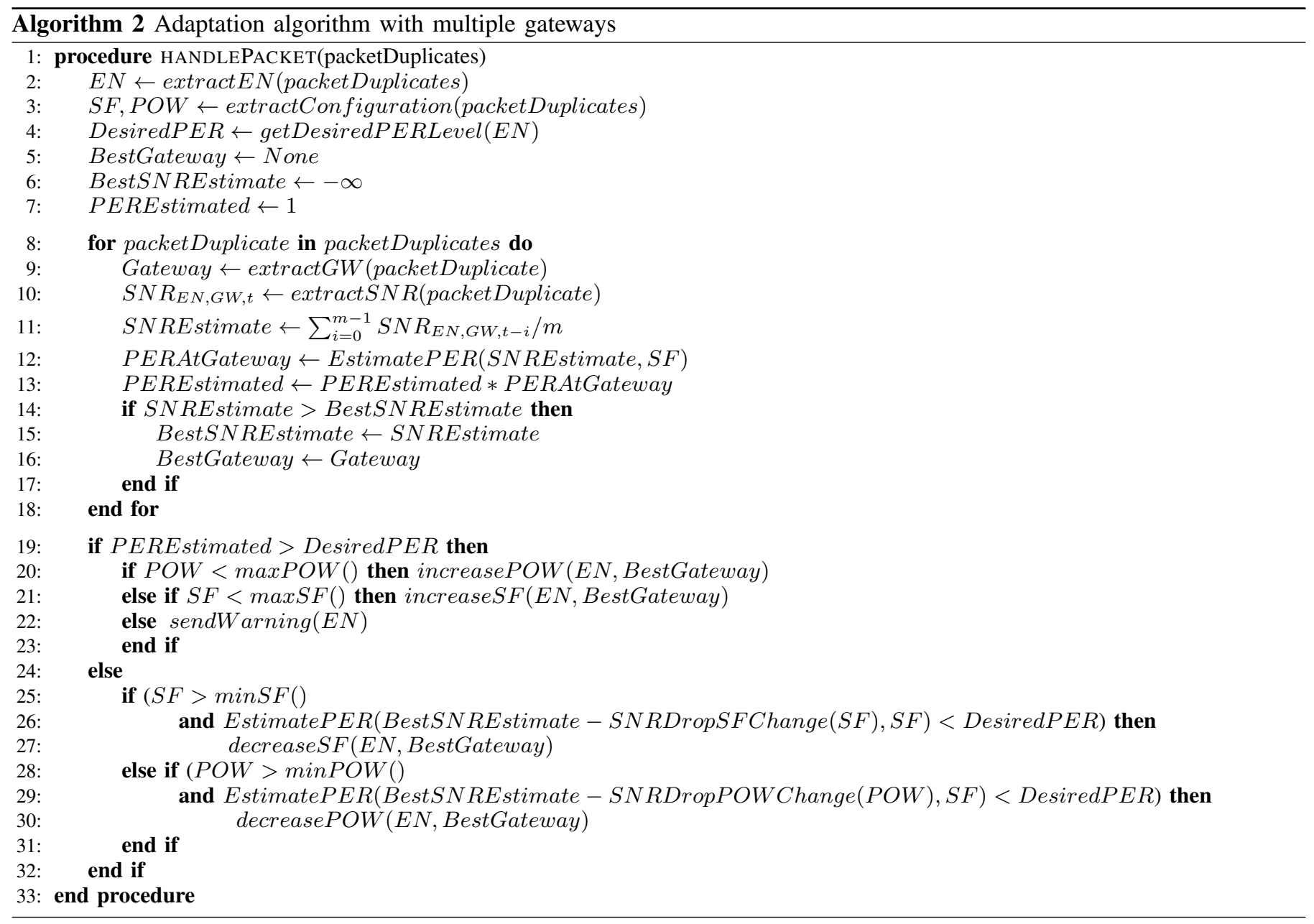

Evaluation with One Gateway. For the evaluation of the algorithm with one gateway, we have set up an experiment as shown in Figure 3 with only GW1 active. During the experiment, the end node starts with moving away from GW1 along the white line over a distance of about $3.2 \mathrm{~km}$ towards GW2. Then the end node returns back to GW1. The goal of the experiment was to demonstrate that the adapation algorithm is capable to dynamically adapt the SF and POW of the mobile end node to achieve the QoS requirements.

Concretely, we used the following communication sequence. The end node sends packets at time intervals of 15 sec. First a baseline packet is sent with a conservative setting (SF 12, POW 15) that would be used without algorithm. Second, the end node sends a packet with the SF and POW settings determined by the algorithm. When the end node receives an adaptation update from the application backend, sent by the gateway, it updates its settings.

Figure 9 shows how the algorithm adapts the node settings during the experiment. Until $\mathrm{km} \mathrm{1.8,} \mathrm{when} \mathrm{the} \mathrm{end} \mathrm{node}$ moves away from GW1 (Figure 9a), a setting SF 10 and POW -3 was used. Between $\mathrm{km} 1.8$ and 3.0, the POW setting was gradually increased from -3 to 6 and 15 (SF was kept constant at 10). At $\mathrm{km} \mathrm{3}$, the algorithm increased the SF to 11. When returning from GW2 to GW1 (Figure 9b), a similar inverse pattern is followed where the settings are gradually adapted from SF 12 and POW 15 back to SF10 and POW -3 .
The adaptations ensure that the end node remains in a connected region throughout the experiment, so PER remains below $10 \%$. The power consumed by the end node is proportional to the SF and POW settings, which are optimized by the algorithm. Table 7 compares the results of the algorithm with the baseline approach. The results show that the algorithm reduces energy consumption (in milliampere * hours) of about $80 \%$, at the cost of a small increase of the packet error rate (which remains at all times below the required 10\%).

\begin{tabular}{|c|c|c|c|}
\hline & Direction & EC (mAh) & PER \\
\hline \multirow{2}{*}{ Algorithm } & Away from GW1 & 0.81 & $1.4 \%$ \\
\cline { 2 - 4 } & Towards GW1 & 0.88 & $6.5 \%$ \\
\hline \multirow{2}{*}{ Baseline } & Away from GW1 & 4.17 & $1.4 \%$ \\
\cline { 2 - 4 } & Towards GW1 & 4.17 & $5.0 \%$ \\
\hline
\end{tabular}

TABLE 7: Results adaptation algorithm versus baseline

Evaluation with Multiple Gateways. For the evaluation of the algorithm with multiple gateways, we used the same setup (Figure 3). An end node moves from GW1 to GW2, now with the two gateways active. The goal of the experiment is to demonstrate that the adaptation algorithm with multiple gateway is able to dynamically adapt the SF and POW of the mobile end node to achieve the QoS requirements by exploiting the data received by the two gateways.

The end node sends packets every $15 \mathrm{sec}$ while moving along the trajectory. Each gateway measures the SNR of the 


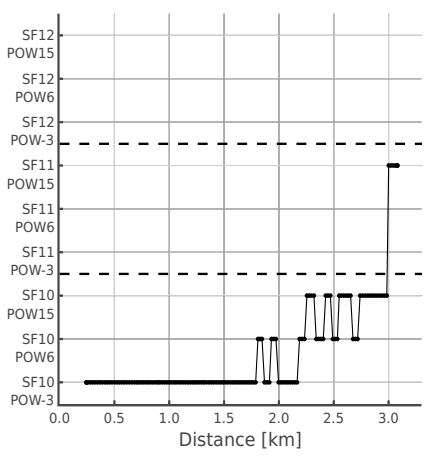

(a)

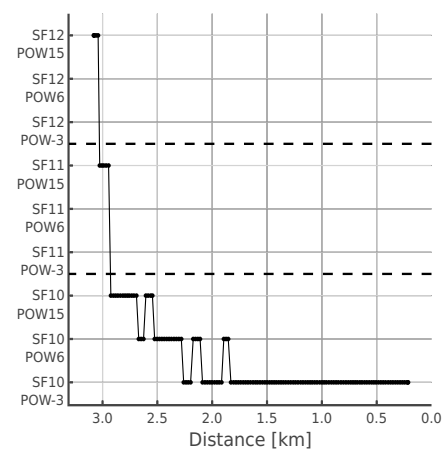

(b)
Figure 9: Dynamic adaptation of SF and POW of a mobile end node using the algorithm with one gateway for: (a) moving away from GW1, and (b) moving towards GW1.

packets it receives. This data is then fed to the algorithm with multiple gateways to determine the resulting PER and EC of the end node and adapt the settings if required (leaving connected region) or possible (entering new connected region).

Figure 10a shows the evolution of the PER for the two gateways when the end node moves along the trajectory using different POW settings. This data would be used if the adaptation decisions would be made by individual gateways. Figure $10 \mathrm{~b}$ shows the combined PER for different POW settings.

Figure 11a shows the results of the algorithm with two gateways applied to the experimental setup. Until $\mathrm{km} \mathrm{2,} \mathrm{the}$ algorithm dynamically adapts the power setting and spreading factor to keep the end node in a connected region. Between $\mathrm{km} 2.0$ and 2.4, the end node reaches it maximum settings (SF 12, POW 15). At that time, there is a risk that the reliability requirement is temporally not achieved. After km 2.4 when the end node approaches GW2, the settings are relaxed again.

Figure $11 \mathrm{~b}$ shows a concrete adaptation scenario for the first $1.8 \mathrm{~km}$ and the last first $0.8 \mathrm{~km}$ of the trajectory. The SF in these parts of the trajectory is set at 10 (see Figure 11a). In the first part, an initial POW of -3 is used. Around km 0.85 , the PER threshold is reached $(0.1$ or $10 \%)$ and the POW is increased to 6 . As a result, the PER drops again to a very low value. The same happens around $\mathrm{km} 1.4$ when the POW is further increased to 15 . Around $\mathrm{km} \mathrm{1.9,} \mathrm{the} \mathrm{PER} \mathrm{reaches} \mathrm{again}$ its threshold. Now the spreading factor is increased to 11 (as the POW setting has reached its limit). A similar pattern can be observed in last part of the trajectory, where the end node dynamically reduces the POW setting from 15 to -3 .

Table 8 compares EC and PER of the algorithm with the baseline approach (SF 12, POW 15). The results show that the algorithm realizes a significant improvement of energy consumption. The cost is a small increase in packet error rate.

\begin{tabular}{|c|c|c|}
\hline & EC (mAh) & PER \\
\hline Algorithm & 1.33 & $3.9 \%$ \\
\hline Baseline & 3.90 & $2.9 \%$ \\
\hline
\end{tabular}

TABLE 8: Algorithm with two gateways versus baseline

Threats to Validity. We evaluated the algorithms for an IoT setup with specific technology, which affects the generalization of the results (external validity). To minimize this threat, we developed the setup and selected the environment in collaboration with VersaSense (www.versasense.com), a

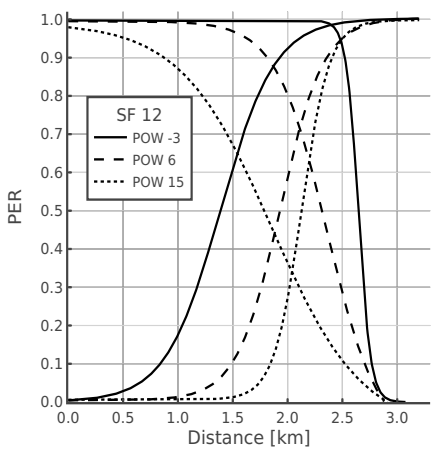

(a)

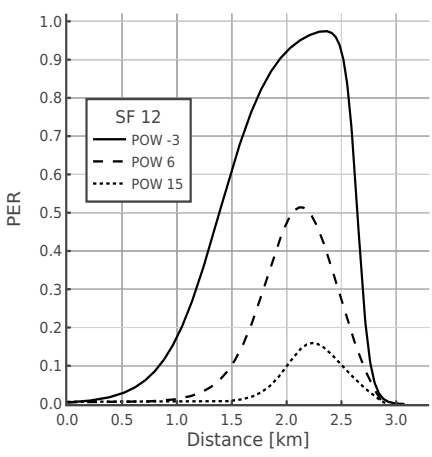

(b)
Figure 10: PER along trajectory for SF 12 and different POW: (a) PER separately per gateway and (b) combined.

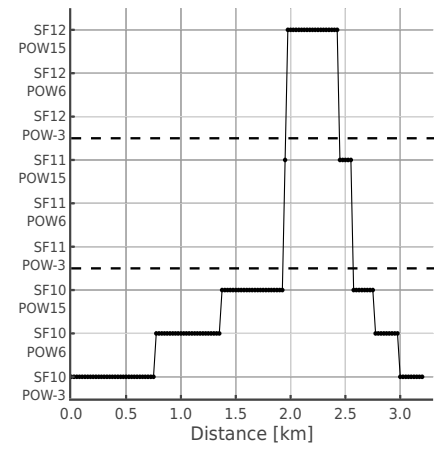

(a)

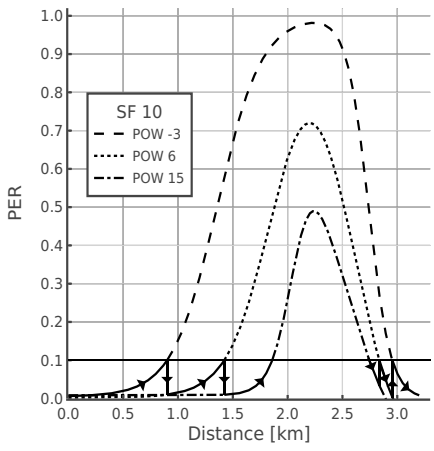

(b)
Figure 11: Adaptation with two gateways: (a) change of end node settings, and (b) concrete adaptation scenario (SF 10).

provider of industrial IoT solutions. This ensures a setup that is representative for practical IoT systems, while keeping the conditions in the environment as stable as possible. Finally, all the data and results are available allowing to repeat or extend the study presented in this paper (reliability). ${ }^{9}$

\section{Related Work}

We discuss related work in two parts. First, we highlight a number of representative approaches that take a software engineering perspective on adaptation in wireless sensor networks and IoT. Second, we zoom on a selection of related work that take a network perspective, in particular LoRa.

QARI [25] uses reconfiguration primitives to ensure that quality of service levels remain intact when operational conditions change in wireless sensor networks. [26] addresses the problem of dynamic changes of feature models in sensor networks product families, where nodes of the network demand dynamic reconfiguration after deployment. [27] show that aggregate programming can dramatically simplify the development and maintenance of complex IoT software systems by leveraging mechanisms that provide robust and adaptive coordination. [28] presents a number of recent developments, including energy and resource management in machine to machine communications and federation of IoT services with $5 \mathrm{G}$. This selection of work shows that adaptation has been applied to IoT. Compared to our work, these approaches

9. https://people.cs.kuleuven.be/danny.weyns/software/LoRa/index.htm 
focus at higher levels of the technology stack. Moreover, most proposed solutions are applied in simulated systems only.

The authors of [29] and [30] compare different long-range technologies, including LoRa. [31] studies the performance of a low-power wide-area network based on LoRa technology, including robustness, scalability, and coverage. [32] analyzes the LoRa capacity and proposed LoRaBlink to support multihop communications. DeltaIoT [33] is a recently developed prototypical IoT system proposed as an artifact for research experimentation on adaption in LoRa. It has been applied for example to investigate a novel cost-benefit adaptation schema for different QoS requirements [34]. Finally, the most closely related work is [4] that performs an in-depth analysis of LoRa. The authors provide a functional description of LoRa, as well as a quantification and evaluation of the performance of LoRA especially with respect to the spreading factor. Complementary to the presented related approaches, this paper focusses in particular on mobility in LoRa networks and provides a concrete solution to exploit the knowledge obtained from empirically collected data to build adaptation solutions to achieve the QoS requirements of LoRa-based IoT systems.

\section{Conclusions and Future Work}

Internet-of-Things are widely considered as a key enablers towards the realisation of smart cities. In this paper, we looked at mobility of end nodes in LoRa-based IoT systems. In order to achieve the QoS requirements in such settings, end nodes need to adapt dynamically their settings. In our work, we put reliability and energy consumption in the spotlight, two key qualities for LoRa-based IoT systems with battery-powered mobile end nodes. We presented the results of extensive experiments that allowed us to determine the key performance indicators to track reliability and energy consumption. We also assessed the parameters that fit best to adapt the settings of the mobile end nodes to obtain the QoS requirements. Based on these insights we contribute an algorithm that is able to adapt the settings of a mobile end node automatically in order to achieve the reliability and energy consumption requirements. We extended the initial version of the algorithm for a single gateway to a general setting with multiple gateways. We demonstrated that the algorithms achieve their goals in a practical IoT deployment. In future research we plan to apply the algorithm to a large scale IoT setup, for which we can use DingNet, a smart city network deployed in the Leuven area. We also plan to study the application of learning techniques to scale solutions. In the long term, we aim at integrating adaption solutions vertically across layers of the technology stack and horizontally across tiers of the IoT system.

\section{References}

[1] N. Heuveldop et al., "Ericsson mobility report," Ericsson AB, Technol. Emerg. Business, Sweden, Tech. Rep. EAB-17, vol. 5964, 2017.

[2] L. Atzori, A. Iera, and G. Morabito, "The Internet of Things: A survey," Computer networks, vol. 54, no. 15, 2010.

[3] A. Whitmore et al., "The Internet of Things: a survey of topics and trends," Information Systems Frontiers, vol. 17, no. 2, 2015.

[4] A. Augustin et al., "A study of LoRa: Long range \& low power networks for the Internet of Things," Sensors, vol. 16, no. 9, 2016.

[5] LoRa-Alliance, "White paper: A technical overview of LoRa and LoRaWAN," 2015, san Ramon, CA, USA.
[6] E. Borgia, "The Internet of Things vision: Key features, applications and open issues," Computer Communications, vol. 54, 2014.

[7] J. Yick, B. Mukherjee, and D. Ghosal, "Wireless sensor network survey," Computer networks, vol. 52, no. 12, 2008.

[8] X. Xiong et al., "Low power wide area machine-to-machine networks: key techniques and prototype," IEEE Communications Magazine, vol. 53, no. 9, 2015

[9] P. Rawat et al., "Wireless sensor networks: a survey on recent developments," The Journal of Supercomputing, vol. 68, no. 1, 2014.

[10] D. Miorandi et al., "Internet of Things: Vision, applications and research challenges," Ad Hoc Networks, vol. 10, no. 7, 2012.

[11] N. Shahid and S. Aneja, "Internet of Things: Vision, application areas and research challenges," in International Conference on IoT in Social, Mobile, Analytics and Cloud, 2017.

[12] D. Weyns, G. S. Ramachandran, and R. K. Singh, "Self-managing Internet of Things," in 44th International Conference on Current Trends in Theory and Practice of Computer Science, 2018.

[13] D. Weyns et al., "Applying architecture-based adaptation to automate the management of internet-of-things," in European Conference on Software Architecture, 2018.

[14] I. F. Akyildiz et al., "Wireless sensor networks: a survey," Computer networks, vol. 38, no. 4, 2002.

[15] C. Bettstetter, "Mobility modeling in wireless networks: categorization, smooth movement, and border effects," Mobile Computing and Communications Review, vol. 5, no. 3, 2001.

[16] J. Petajajarvi et al., "On the coverage of LPWANs: range evaluation and channel attenuation model for lora technology," in International Conference on ITS Telecommunications. IEEE, 2015.

[17] L. Li, J. Ren, and Q. Zhu, "On the application of LoRa LPWAN technology in sailing monitoring system," in Annual Conference on Wireless On-demand Network Systems and Services. IEEE, 2017.

[18] M. Aref and A. Sikora, "Free space range measurements with Semtech LoRa technology," in Wireless Systems. IEEE, 2014.

[19] N. Baccour et al., "Radio link quality estimation in wireless sensor networks: A survey," Trans. on Sensor Networks, vol. 8, no. 4, 2012.

[20] P. Oreizy et al., "An architecture-based approach to self-adaptive software," IEEE Intelligent Systems, vol. 14, no. 3, pp. 54-62, 14(3), 1999.

[21] R. De Lemos et al., "Software engineering for self-adaptive systems: Research challenges in the provision of assurances," in Software Engineering for Self-Adaptive Systems III. Springer, 2017.

[22] D. Weyns, "Software Engineering of Self-Adaptive Systems: An Organised Tour and Future Challenges," Chapter in Handbook of Software Engineering, Springer, 2018.

[23] D. Weyns, N. Bencomo, R. Calinescu et al., "Perpetual Assurances in Self-Adaptive Systems," in Software Engineering for Self-Adaptive Systems, ser. Lecture Notes in Computer Science, Vol 9640, 2018.

[24] D. Weyns et al., "Do External Feedback Loops Improve the Design of Self-Adaptive Systems? A Controlled Experiment," in SEAMS, 2013.

[25] W. Horr et al., "QARI: Quality aware software deployment for wireless sensor networks," in Information Technology: New Generations, 2010.

[26] O. Ortiz et al., "Runtime variability for dynamic reconfiguration in wireless sensor network product lines," in International Software Product Line Conference - Volume 2. ACM, 2012.

[27] J. Beal, D. Pianini, and M. Viroli, "Aggregate programming for the internet of things," Computer, vol. 48, no. 9, 2015.

[28] E. Natalizio et al., "Advances in wireless communication for cooperating autonomous systems," Ad Hoc Networks, vol. 68, no. C, 2018.

[29] N. Rathod et al., "Performance analysis of wireless devices for a campus-wide IoT network," in International Symposium on Modeling and Optimization in Mobile, Ad Hoc, and Wireless Networks, 2015.

[30] M. Centenaro et al., "Long-range communications in unlicensed bands: the rising stars in the IoT and smart city scenarios," IEEE Wireless Communications, vol. 23, no. 5, 2016.

[31] J. Petäjäjärvi et al., "Performance of a low-power wide-area network based on LoRa technology: Doppler robustness, scalability, coverage," Journal of Distributed Sensor Networks, vol. 13, no. 3, 2017.

[32] M. Bor et al., "LoRa for the Internet of Things," in Embedded Wireless Systems and Networks, 2016.

[33] M. U. Iftikhar et al., "DeltaIoT: A self-adaptive Internet of Things exemplar," in SEAMS, 2017.

[34] J. Van Der Donckt et al., "Cost-benefit analysis at runtime for selfadaptive systems applied to an iot application," in ENASE, 2018. 